\title{
1 Strategies for targeting BAT thermogenesis
}

2 Andrew Whittle ${ }^{1 *}$, Joana Relat-Pardo $^{2}$, Antonio Vidal-Puig ${ }^{1 *}$

$3 \quad{ }^{1}$ Institute of Metabolic Science, University of Cambridge, Cambridge, UK, CB2 0QQ

$4 \quad{ }^{2}$ Department of Biochemistry and Molecular Biology, School of Pharmacy and the

5 Institute of Biomedicine of the University of Barcelona (IBUB), University of

6 Barcelona, Barcelona, Spain, 08028

7

8 * Corresponding authors:

9 University of Cambridge Metabolic Research Laboratories

10 Level 4, Institute of Metabolic Science

11 Box 289, Addenbrooke's Hospital

12 Cambridge, CB2 0QQ

13 United Kingdom

14 Tel: +44 1223769095

15 Fax: +44 1223330598

16 ajw232@medschl.cam.ac.uk

17 ajv22@cam.ac.uk

18

19 
21 Modern imaging techniques have confirmed the presence of thermogenically active brown adipose tissue (BAT) in adult humans, leading to suggestions that it could be stimulated to treat obesity and its associated morbidities. The mechanisms regulating thermogenesis in BAT are better understood than ever before, with new hypotheses for increasing the amount of brown fat or its activity being put forward on a weekly basis. The challenge now is to identify safe ways to manipulate specific aspects of the physiological regulation of thermogenesis, in a manner that will be bioenergetically effective. This review outlines the nature of these regulatory mechanisms both terms of their cellular specificity and likely effectiveness given the physiological paradigms in which thermogenesis is activated. Similarly, their potential for being targeted by new or existing drugs is discussed, drawing on the known mechanisms of action of various pharmacological agents and some likely limitations that should be considered.

\section{Introduction}

By no means is brown adipose tissue (BAT) a novel discovery; the "hibernating gland" was first described in rodents by the Swiss naturalist Conrad Gessner as early as $1551^{1}$. In more recent times throughout the $20^{\text {th }}$ Century it has been the focus of multiple waves of scientific study, each of which waned as BAT's relevance to adult

39 humans was called into question, largely due to limitations in our ability to measure 40 its presence or activity. The recent resurgence in interest stems from data provided by modern PET-CT imaging studies firmly demonstrating that healthy adults do indeed

42 possess significant depots of BAT ${ }^{2-5}$. Moreover, amounts of detectable, active BAT 43 are inversely correlated with age, BMI, fat mass and insulin sensitivity ${ }^{6}$. At the same 44 time the increased availability of genetically modified murine models has shone more 
45 light than ever before on the endogenous mechanisms that exist to regulate BAT

46 function ${ }^{7}$. Attention has now returned to how we might exploit this tissue more

47 effectively and whether any of these regulatory systems lend themselves to

48 pharmacological or therapeutic intervention. It is posited that increasing the

49 prevalence and activity of BAT might be an effective strategy to treat conditions such

50 as obesity, diabetes and cardiovascular disease, via increased energy expenditure, the

51 removal of toxic lipid species and a direct reduction in the demand for insulin

52 production. This review will focus on the latest knowledge surrounding BAT

53 regulation and highlight potential approaches to target thermogenesis safely and

54 effectively. It will also comment on situations where dysregulation of BAT may

55 contribute to certain metabolic diseases.

57 The master regulators of BAT, lest we forget

58 While the views towards BAT's prevalence and relevance have changed dramatically

59 over recent decades one fact has remained widely undisputed. BAT is under the direct

60 regulation of the sympathetic nervous system, the tone of which to BAT is increased

61 by exposure to a cold environment or in response to increased caloric intake.

62 Subsequent activation of various isoforms of the $\beta$-adrenergic receptors by

63 noradrenaline to induce both the thermogenic activity of existing brown adipocytes

64 and the recruitment of new cells to BAT depots is therefore essential for heat

65 production ${ }^{8}$. Similarly, intact thyroid hormone signalling is crucial for thermogenesis

66 in BAT, signified by two key observations. Circulating thyroid hormone and BAT

67 expression levels of the thyroid hormone activating enzyme deiodinase II (DioII) are

68 strongly increased in situations of increased thermogenesis and genetic ablation of

69 DioII in BAT renders animals incapable of adaptive thermogenesis ${ }^{9}$. Therefore when 
considering strategies to increase BAT thermogenesis we should be mindful of the necessity to coincidently increase or at least maintain tonic levels of BAT activation by these endogenous regulatory mechanisms. Failure to do so will likely negate the efficacy of approaches targeting more specific aspects of brown adipocyte biology.

\section{Increasing the amount of BAT}

To understand how to increase the amount of BAT, we must first learn where it comes from. In mouse models, cell lineage tracing has shown fairly conclusively that in development brown adipocytes arise from an origin distinctly different to that of white adipocytes. Brown adipocytes arise from a cell lineage expressing the myogenic gene $M y f 5$, which are also able to differentiate into skeletal muscle cells ${ }^{10}$. In BAT the induction of key transcription factors leads to repression of myogenic gene expression and facilitates brown fat determination. In the last 5 years a number of these factors, PR domain contain 16 (PRDM16), Sirtuin 1 (SIRT1) and the signals that can induce them, such as bone morphogenetic protein 7 (BMP7) have begun to be identified ${ }^{10-12}$. Despite their different origins, all adipose tissues are dependant on certain fundamental transcriptional regulators to drive preadipocyte cells towards mature adipocytes, namely peroxisome proliferator-activated receptor-gamma $88(\mathrm{PPAR} \gamma)$ and CCAAT/enhancer-binding proteins (C/EBPs) ${ }^{13}$. Expression of just

$89 \mathrm{C} / \mathrm{EBP} \beta$, the C/EBP more highly expressed in BAT, and PRDM16 in either human or 90 mouse skin fibroblasts is sufficient to drive these precursors to differentiate into cells

91 displaying the key characteristics of brown adipocytes (expression of uncoupling 92 protein 1, lipid accumulation, responsiveness to cAMP). What is more, when 93 transplanted back into mice these genetically modified fibroblasts formed an ectopic 
94 fat pad that appeared and behaved similarly to BAT, taking up labelled glucose to give a positive PET-CT signal ${ }^{14}$. our perception of our environmental temperature. In the aforementioned study by Kajimura et al. the modified BAT-recipient mice were housed at room temperature $\left(20{ }^{\circ} \mathrm{C}-23{ }^{\circ} \mathrm{C}\right)$, a sub-thermoneutral temperature for animals of their size.

101 Thermogenic demand would have thus been reasonably high such that they were driven to activate their additional pool of new BAT. The same temperature requires no additional heat productions for humans to maintain their core temperature and therefore very little sympathetic activation of thermogenesis. Given the importance of

105 adrenergic tone to BAT it is likely that a similar study in humans would have simply 106 seen the extra thermogenic capacity of the transplanted cells largely or even 107 completely unutilised. That said, the study does demonstrate that population of 108 modified adipocytes, under the right environmental setting can engraft and acquire

109 adequate vascularisation and innervation to become metabolically active to a 110 significant degree. Also, whilst GM-cell transplants are currently some way off use in

111 humans they do provide a potential future approach to avoid deleterious off-target 112 effects, as to date very little is known about the effects of factors such as PRDM16 in 113 non-adipose tissues.

\section{Increasing the number of "beige" adipocytes}

116 When mice are exposed to colder environmental temperatures and adaptive 117 thermogenesis is activated, increased numbers of "brown-like" or "beige" adipocytes 118 appear in traditional white adipose tissue depots. These cells are unique in the sense 
119 that they express all of the thermogenic machinery but not Myf5, indicating they arise 120 from a different developmental origin to canonical BAT ${ }^{15}$. The same effect is seen in

121 animals treated with agonists of PPAR $\gamma^{16}$ and interest in these cells has grown for a 122 two key reasons. First, strains of mice that have a higher propensity for "browning" of 123 their white fat show a greater degree of protection from diet-induced obesity when 124 treated with $\beta 3$-adrenergic receptor agonists ${ }^{17}$ (this is the main receptor isoform 125 regulating thermogenic activity in BAT); and second, it has recently been suggested 126 that the gene expression profile of adult human BAT bears greater resemblance to that 127 of beige adipocytes than murine brown adipocytes found in traditional interscapular 128 depots ${ }^{18}$. This finding is hugely encouraging, as traditional BAT depots decline 129 rapidly in childhood and into adolescence, leaving only small residual repositories of 130 thermogenically active tissue by post-pubertal ages ${ }^{19}$. In adult mice beige adipocytes 131 require active induction and recruitment under specific environmental conditions, 132 therefore these human observations at very least suggest that such "browning" 133 mechanisms might remain intact and effective at maintaining a population of 134 thermogenic cells well into later life.

136 When seeking to increase the pool of beige adipocytes in white adipose tissue (WAT) 137 we may only have to look as far as skeletal muscle. It had been observed that exercise 138 was sufficient to increase the expression of thermogenic genes in WAT whilst at the 139 same time protecting mice from the deleterious effects of a high fat diet ${ }^{20}$. 140 Subsequently an exercise-induced myokine, namely irisin, has been characterised 141 with the ability to induce a thermogenic program of gene expression in white adipose 142 tissue, increase energy expenditure and reduce rates of weight gain in mice ${ }^{21}$. At 143 present this finding should encourage people of the multitude of benefits resulting 
144 from increased exercise. However, before irisin is hailed as "magic switch" for

145 turning white fat brown, a couple of important points must be addressed. At present

146 the irisin receptor remains elusive and the effects of irisin in other crucial organs have

147 not been examined. A molecule that integrates increased muscle and thermogenic

148 function may also be expected to act on the heart to meet the extra requirements of

149 oxygen provision. In obese individuals already carrying an increased cardiovascular 150 burden, such effects may not be well tolerated.

152 Other avenues for increasing beige adipocyte content do exist. MicroRNA-133 153 (miRNA-133) is highly expressed in skeletal muscle and is heavily downregulated in 154 brown and white adipose depots in response to cold-exposure in mice. Further studies 155 show that miRNA-133 directly targets PRDM16 to repress its browning effects in 156 adipose tissue and that inhibition of miRNA in BAT or WAT precursor cells allows 157 increased expression of thermogenic genes and increased oxidative metabolism ${ }^{22}$.

158 Given that pharmacological strategies that suppress individual miRNAs have already 159 been tested with some success in humans to treat hepatitis ${ }^{23}$, this strategy may also 160 show promise for BAT-centric, anti-obesity therapy. As always there is air for caution. 161 miRNA-133 has recently been identified as an important tumour-suppressor ${ }^{24}$, while 162 its downregulation associates with cardiac hypertrophy and infarction ${ }^{25,26}$, perhaps 163 making global pharmacological repression of its expression less than favourable.

\section{Increasing BAT activity}

166 As alluded to earlier in this review, it is likely of limited benefit to provide an 167 additional pool of thermogenic cells to an individual unless the physiological setting 168 is such that they will receive at least a minimal level of stimulation from the 
sympathetic nervous system. Similarly, the efficacy of any BAT-expanding approach

170 is likely to be enhanced by parallel strategies to enhance adrenergic stimulation. To

171 this end bioactive food ingredients such as methylxantines (caffeine or theophylline),

172 ephedrine, polyphenols (catechins, resveratrol, querectin, kaempferol) and certain

173 fatty acids or drugs such as sibutramine, which increase or maintain sympathetic

174 nervous system activation, are effective at increasing energy expenditure and

175 lowering body weight ${ }^{27-29}$. However, the fact that the effects of sibutramine and other

176 sympathomimetics cannot be targeted to a specific tissue has rendered them unsafe for

177 use in humans ${ }^{30}$.

179 Increasingly researchers are attempting to define more specific physiological 180 mechanisms that regulate the response of BAT to endogenous levels of sympathetic 181 stimulation, with some success. An increasing number of cytokines and hormones 182 have been shown to have positive effects on the thermogenic activity of BAT and 183 perhaps most interestingly, none of them appear to act via the adrenergic receptors 184 directly but instead through more specific aspects of brown adipocyte biology.

FGF (fibroblast growth factor) 21 is secreted predominantly by the liver, where levels 187 rise during fasting, feeding a ketogenic diet or after amino acid deprivation; but also 188 by other tissues such as WAT, BAT, skeletal muscle and pancreatic $\beta$-cells following 189 different metabolic signals ${ }^{31,32}$. FGF21 signals to BAT to increase expression of 190 thermogenic genes and enhance its heat-producing capacity and regulates the 191 browning of white adipose tissue though PGC1 $\alpha$ signalling ${ }^{33-35}$. In line with this, 192 treatment of obese mice with FGF21 is sufficient to reduce bodyweight by $20 \%$, 193 almost entirely due to increased energy expenditure ${ }^{36}$. Paradoxically, circulating 
194 levels of FGF21 increase in obesity in humans. However further investigations reveal 195 that the same is true in mice and that treatment of obese mice with additional FGF21 196 brings about markedly reduced activation of FGF21 targets, such as ERK1/2. In light 197 of this, obesity has been suggested to constitute a state of FGF21 resistance, which 198 may go some way to contribute to obesity-associated metabolic complications and 199 perpetuate a state of positive energy balance ${ }^{37}$.

201 Although the intracellular pathways activated by FGF21 in BAT are unclear its 202 membrane interaction is well characterised. FGF21 signals through traditional FGF 203 receptors conjugated to the membrane protein $\beta$ Klotho ${ }^{38}$, which may offer 204 opportunities to target the thermogenic properties of FGF21 more selectively of other 205 growth factor-activated pathways.

207 We have recently characterised bone morphogenetic protein 8b (BMP8B) as another 208 thermogenically regulated protein, enriched in mature brown adipocytes in line with 209 their level of thermogenic activity ${ }^{39}$. BMP8B is a secretory peptide that acts in an 210 autocrine/paracrine manner to increase the thermogenic capacity of BAT by 211 enhancing the intracellular response to adrenergic stimulation. Mice that lack BMP8B 212 have lower core body temperatures, reduced thermogenic capacity and are 213 significantly more susceptible to diet-induced obesity due to a reduction in their 214 metabolic rate. Any ability to modulate adrenergic sensitivity in a tissue specific 215 manner is likely to be of great potential benefit for the treatment of a range of 216 conditions in addition to obesity, such as hypertension and various forms of fibrotic 217 liver disease. Whilst the precise mechanisms of BMP8b remains unclear, its distinct 218 expression profile of and the high degree of cellular specificity in the BMP receptor 
system, recently reviewed by Sieber et al ${ }^{40}$, make it a promising future target for activating BAT independently of other sympathetically regulated organs.

222 Of perhaps even greater interest is the fact that a significant portion of the 223 thermogenic effect of BMP8B can likely be attributed to its central role. ICV 224 treatment with BMP8b results in an immediate increase in sympathetic tone to BAT 225 and this effect is dependant on the level of AMP-activated protein kinase (AMPK) in 226 the ventromedial nucleus of the hypothalamus, a well defined regulatory node for 227 thermogenic activation ${ }^{41}$. Whilst the global role of AMPK in cellular bioenergetics 228 would appear to make it a difficult target when aiming to selectively activate BAT, 229 these findings to highlight that there are discrete nuclei within the central circuits 230 regulating BAT that might one day be amenable to therapeutic manipulation. The fact 231 that BMP8B may be an endogenous activator of such nuclei makes dissecting its 232 mechanism of action all the more important. Already it has been shown that blockade 233 of an individual BMP receptor, Activin Receptor IIB, induces thermogenesis and 234 brown adipogenesis ${ }^{42}$.

\section{Targeting central regulation of BAT}

237 Our understanding of the central control of thermogenesis has been expanded vastly

238 in the last decade. This is thanks largely to detailed electrophysiological investigations

239 of specific neuronal population by Morrison and Madden, which are the focus of 240 recent review ${ }^{43}$. In summary, classical cold-activated thermogenesis is brought about 241 when cold sensitive temperature receptors in the skin signal to the pre-optic area of 242 the hypothalamus (POA). Activation of these GABA-ergic neurons disinhibits 243 neurons of the dorsomedial hypothalamus (DMH), which are themselves suppressed 
244 by signals from warm-sensitive temperature receptors. Subsequently projections from

245 the DMH activate sympathetic pre-motor neurons in the rostral raphe pallidus of the

246 hindbrain (rRPA), which signal to BAT to increase thermogenesis. Certain

247 nutritionally-derived molecules are known to act as agonists for various isoforms of

248 these temperature receptors, offering a means to activate the endogenous machinery at

249 the most upstream point.

251 Capsaicin, the compound found in hot chilli, acts a ligand for the transient receptor 252 potential cation channel subfamily V member 1 (TRPV1). This receptor is highly 253 expressed in sensory neurons and is stimulated by intense heat ${ }^{44}$. Seemingly 254 paradoxically, treatment of rats with capsaicin is known to actually increase activation 255 of thermogenesis ${ }^{45}$ and more recently capsaicin has been shown to cause similar 256 effects in humans ${ }^{46}$. Whilst this raises the possibility of nutritionally-mediated 257 increases to energy expenditure to treat obesity the studies performed to date have 258 only been conducted in the acute setting. The rat studies indicate some level of 259 protection from diet induced obesity but it should be noted that animals of this size 260 already have good levels of thermogenic activation, and indeed the capsaicin had no 261 effects in BAT-negative individuals.

263 Menthol is also able to stimulate thermogenesis through a separate temperature 264 receptor, TRP subfamily $\mathrm{M}$ member 8 (TRPM8). TRPM8 is also expressed in the 265 sensory and central nervous system, as well as on brown adipocytes themselves ${ }^{47}$. 266 Menthol treatment of mice is also effective at protecting them from diet-induced 267 obesity through increased thermogenesis, but a portion of this effect can be attributed 268 to direct effects on BAT itself. TRPM8 treatment of brown adipocytes increases 
expression of UCP1 via a mechanism not hugely dissimilar to BMP8B, whereby the

270 phosphorylation status of key intracellular kinases, in this case PKA, is also increased.

271 It should still be considered however that activation of traditional cold-induced

272 thermogenesis is known to have a potent positive effect on food intake ${ }^{8}$, potentially

273 limiting its long terms effects on energy balance in humans.

\section{Additional central effectors of BAT thermogenesis and the link with nutrition}

In addition to cold, increasing caloric intake via western or high fat diets also increases heat production in BAT, via a process termed diet-induced thermogenesis ${ }^{48}$.

278 As yet the central mechanisms driving this phenomenon is unknown, but there is an 279 ever increasing number of neuroactive molecules that could be involved in feedingrelated changes to sympathetic outflow to BAT. Such mechanisms are of interest for two reasons. First, in situation where food intake is the signal for activating 282 thermogenesis, it is unlikely further food intake would be stimulated as is the case in 283 cold-stimulated thermogenesis. Therefore mimicking these signals may be more 284 efficacious for weight loss. Second understanding the interaction between 285 thermogenesis and feeding may open the door for inhibiting compensatory food 286 intake when pharmacologically mimicking cold-stimulated thermogenesis.

287 Appetite-regulating molecules released by the digestive system (such as ghrelin and 288 calcitonin gene-related peptide) have documented effects on BAT activity ${ }^{49-51}$. 289 Equally, changes in nutrient availability might be directly sensed by the brain. 290 Specific hypothalamic neurons including orexin/hypocretin neurons are sensitive to 291 nutrients (including glucose, amino acids, and fatty acids), and glucose and lipid 292 species can act centrally to activate thermogenesis ${ }^{52,53}$. 
294 The inclusion of the hypocretin system and also the dopaminergic and serotonergic systems ${ }^{54}$ in the list of central BAT-regulatory mechanisms suggests that there may also be links between thermogenesis and arousal status, reward and stress. Further

297 evidence for a link between more complex behavioural traits and thermogenesis may

298 be inferred from the observation that in sufferers of anorexia, treatment to induce 299 weight gain is often hindered by inappropriately high levels of feeding-induced 300 thermogenesis ${ }^{55}$.

\section{Antipsychotics, body weight and BAT}

303 It is well documented that certain atypical antipsychotics lead to increased weight 304 gain in both humans and rodents ${ }^{56}$. Closer examination of the known mechanisms of 305 action of these drugs can shed light on a potential link to altered thermogenesis in BAT. Schizophrenia is largely characterised as a state of dopamine receptor 307 supersensitivity and indeed the efficacy of different antipsychotics correlates with their ability to bind and antagonise dopamine $\mathrm{D}_{2}$ receptors ${ }^{57}$. Olanzapine for example 309 is a $D_{2}$ antagonist which causes weight gain but importantly it is a far more potent

310 inhibitor of serotonergic signaling through its antagonism of various 5-HT receptor

311 isoforms ${ }^{58}$. Recently it has been shown that chemical inhibition of these same 312 receptor subtypes has a dramatic effect of body temperature, significantly reducing it by blocking nervous stimulation of BAT ${ }^{59}$.

315 In this sense one might envisage that selective serotonin reuptake inhibitors (SSRIs) 316 could help to induce the opposite effects. Indeed early studies in humans did indicate 317 weight loss in patients taking SSRIs for relatively short periods, but this was apparently driven largely by reduced food intake ${ }^{60}$. Sibutramine on the other hand, 
which is also a noradrenaline reuptake inhibitor, does have a positive effect on energy expenditure and BAT activity in humans ${ }^{61}$. Taken together these finds suggest that serotonin acts further downstream in thermogenic signalling in coordination with norepinephrine and that at a hypothalamic level its modulation has more potent effects on appetite. It also highlights the inherent complexity of the multiple levels of interaction between energy expenditure and intake.

\section{Increasing the contribution of muscle to thermogenesis}

327 Like BAT, skeletal muscle is well innervated and responds positively to noradrenaline 328 levels through $\beta 3$ - and $\beta 2$-adrenergic receptors. In skeletal muscle, heat production 329 occurs through shivering and non-shivering mechanisms. As shivering is a 330 mechanical muscle response and cannot be sustained for long time the main target 331 processes to sustain prolonged thermogenesis are: mitochondrial uncoupling (as in 332 BAT), futile calcium cycling, and fatty acid/triglyceride cycling that has been shown 333 to be increased following mild-cold exposure ${ }^{62,63}$.

335 Whilst the functional capacity for myocytes to generate heat through uncoupling is 336 still a matter of debate, an adipocyte progenitor population in human skeletal muscle 337 tissue has been described with a high potential for inducing UCP-1 expression. It has 338 been suggested that these cells explain the small amount of UCP1 mRNA often 339 detected in adult human skeletal muscle and that under the relevant conditions they

340 could contribute significantly to energy expenditure. In this instance, part of the heat

341 generated by uncoupling in skeletal muscle may be due to this quiescent brown cell 342 population ${ }^{64}$, which may be amenable to enhancement by the same strategies 343 designed to target BAT directly. 
345 Futile calcium cycling has also been suggested as a potential mechanism to increase

346 skeletal muscle heat production. Here, calcium $\left(\mathrm{Ca}^{2+}\right)$ released into the cytoplasm is 347 recovered to the sarcoplasmic reticulum by SERCA proteins (sarco/endoplasmic 348 reticulum $\mathrm{Ca}^{2+}$-ATPase), which use ATP. In some cases, as in human malignant 349 hyperthermia, there is an uncontrolled leakage of $\mathrm{Ca}^{2+}$ that stimulates calcium cycling 350 without muscle contraction, producing heat ${ }^{65}$. In line with this cold-exposure induces 351 expression and activity of SERCA, and in UCP1 deficient mice leptin-induced 352 thermogenesis increases Serca-2a expression to maintain core body temperature ${ }^{66,67}$.

354 More recently, Sarcolipin has been implicated in skeletal muscle thermogenesis by its 355 role controlling calcium cycling through a direct interaction with SERCA. Sarcolipin 356 deficient mice are more sensitive to cold-exposure, and to a high fat diet (having 357 increased adiposity and glucose intolerant). In terms of adaptive thermogenesis the 358 Sarcolipin-SERCA interaction might be exploited to enhance nonshivering heat 359 production in animals with reduced (humans) or non-functional BAT (pigs) activity ${ }^{68}$,

$360{ }^{69}$. Although more studies need to be done to evaluate the impact of Sarcolipin361 SERCA modulation in human skeletal muscle and heart, this mechanism may open 362 new targets to increase thermogenesis in skeletal muscle by modulating $\mathrm{Ca}^{2+}$ cycling.

\section{Summary}

365 There are a constantly expanding number of regulatory nodes and pathways that 366 integrate BAT function with global physiological changes to ensure its tight 367 regulation. This is to be expected, as the capacity for BAT to "waste" energy is huge. 368 As we learn more about the endogenous molecules and neuronal systems that control 
369 thermogenesis, we are beginning to identify key points that might be amenable to manipulation and thus allow us to disconnect BAT from the tight leash on which it is

371 kept. It may also be true that metabolic changes that occur in certain disease states are 372 disproportionately inhibiting thermogenesis. In either situation identifying the 373 molecular pathways that bring about changes to BAT function is likely to offer 374 therapeutic opportunities. There are already suggestions that increasing levels of key 375 endogenous molecules, such as irisin and FGF21, might be metabolically beneficial in 376 obese states and there is no shortage of receptors to hunt for that likely bring about the 377 thermogenic effects of some of the other molecules described in this review.

379 Key to the medicinal utilisation of BAT thermogenesis, as with any intervention, will 380 be specificity and efficacy. Thankfully it seems that the unique qualities of brown 381 adipocytes bring with them some unique regulatory systems, which may help address 382 the former of the problems. More difficult will be overcoming the complex central 383 regulatory mechanisms that sense heat production and modulate sympathetic nervous 384 stimulation of BAT accordingly. Still it seems that even in the heterogeneity of neural 385 networks there may lie key nuclei that integrate information on temperature and 386 energy availability that might be specifically targeted to alter their level of control 387 over BAT activation. What is almost certain, is that approaches mindful of the 388 multiple levels of regulation of thermogenesis in BAT are likely to be the most 389 effective. 


\section{References}

394 1. Gessner, C. (1551) Conradi Gesneri medici Tigurine Historiae Animalium: 395 Lib. I De Quadrupedibus viviparis.

396 2. Cypess, A.M., et al. (2009) Identification and importance of brown adipose 397 tissue in adult humans. N Engl J Med 360, 1509-1517

398 3. van Marken Lichtenbelt, W.D., et al. (2009) Cold-activated brown adipose 399 tissue in healthy men. $N$ Engl J Med 360, 1500-1508

400 4. Virtanen, K.A., et al. (2009) Functional brown adipose tissue in healthy 401 adults. $N$ Engl J Med 360, 1518-1525

402 5. Zingaretti, M.C., et al. (2009) The presence of UCP1 demonstrates that 403 metabolically active adipose tissue in the neck of adult humans truly represents 404 brown adipose tissue. FASEB J 23, 3113-3120

405 6. Ouellet, V., et al. (2011) Outdoor Temperature, Age, Sex, Body Mass Index, 406 and Diabetic Status Determine the Prevalence, Mass, and Glucose-Uptake Activity 407 of 18F-FDG-Detected BAT in Humans. J Clin Endocrinol Metab 96, 192-199

408 7. Whittle, A. (2012) Searching for ways to switch on brown fat: are we 409 getting warmer? J Mol Endocrinol 49, R79-87

410 8. Cannon, B., and Nedergaard, J. (2004) Brown adipose tissue: function and 411 physiological significance. Physiol Rev 84, 277-359

412 9. Christoffolete, M.A., et al. (2004) Mice with targeted disruption of the Dio2 413 gene have cold-induced overexpression of the uncoupling protein 1 gene but fail 414 to increase brown adipose tissue lipogenesis and adaptive thermogenesis. 415 Diabetes 53, 577-584 
416 10. Timmons, J.A., et al. (2007) Myogenic gene expression signature

417 establishes that brown and white adipocytes originate from distinct cell lineages.

418 Proc Natl Acad Sci U S A 104, 4401-4406

419 11. Seale, P., et al. (2008) PRDM16 controls a brown fat/skeletal muscle $420 \quad$ switch. Nature $454,961-967$

421 12. Tseng, Y.H., et al. (2008) New role of bone morphogenetic protein 7 in 422 brown adipogenesis and energy expenditure. Nature 454, 1000-1004

423 13. Farmer, S.R. (2006) Transcriptional control of adipocyte formation. Cell $424 \quad$ Metab 4, 263-273

425 14. Kajimura, S., et al. (2009) Initiation of myoblast to brown fat switch by a 426 PRDM16-C/EBP-beta transcriptional complex. Nature 460, 1154-1158

427 15. Petrovic, N., et al. (2010) Chronic peroxisome proliferator-activated 428 receptor gamma (PPARgamma) activation of epididymally derived white 429 adipocyte cultures reveals a population of thermogenically competent, UCP1430 containing adipocytes molecularly distinct from classic brown adipocytes. J Biol $431 \quad$ Chem 285, 7153-7164

432 16. Crossno, J.T., Jr., et al. (2006) Rosiglitazone promotes development of a 433 novel adipocyte population from bone marrow-derived circulating progenitor 434 cells. J Clin Invest 116, 3220-3228

435 17. Collins, S., et al. (1997) Strain-specific response to beta 3-adrenergic 436 receptor agonist treatment of diet-induced obesity in mice. Endocrinology 138, $437 \quad 405-413$

438 18. Wu, J., et al. (2012) Beige adipocytes are a distinct type of thermogenic fat 439 cell in mouse and human. Cell 150, 366-376 
440 19. Symonds, M.E., et al. (2012) Thermal Imaging to Assess Age-Related

441 Changes of Skin Temperature within the Supraclavicular Region Co-Locating

442 with Brown Adipose Tissue in Healthy Children. J Pediatr 161, 892-898

443 20. Xu, X., et al. (2011) Exercise ameliorates high-fat diet-induced metabolic

444 and vascular dysfunction, and increases adipocyte progenitor cell population in

445 brown adipose tissue. Am J Physiol Regul Integr Comp Physiol 300, R1115-1125

446 21. Bostrom, P., et al. (2012) A PGC1-alpha-dependent myokine that drives

447 brown-fat-like development of white fat and thermogenesis. Nature 481, 463-

$448 \quad 468$

449 22. Trajkovski, M., et al. (2012) MyomiR-133 regulates brown fat

450 differentiation through Prdm16. Nat Cell Biol 14, 1330-1335

451 23. Lindow, M., and Kauppinen, S. (2012) Discovering the first microRNA452 targeted drug. J Cell Biol 199, 407-412

453 24. Moriya, Y., et al. (2011) Tumor suppressive microRNA-133a regulates 454 novel molecular networks in lung squamous cell carcinoma. J Hum Genet 57, 38$455 \quad 45$

456 25. Care, A., et al. (2007) MicroRNA-133 controls cardiac hypertrophy. Nat 457 Med 13, 613-618

458 26. Bostjancic, E., et al. (2010) MicroRNAs miR-1, miR-133a, miR-133b and 459 miR-208 are dysregulated in human myocardial infarction. Cardiology 115, 163$460 \quad 169$

461 27. Dulloo, A.G. (2011) The search for compounds that stimulate 462 thermogenesis in obesity management: from pharmaceuticals to functional food 463 ingredients. Obes Rev 12, 866-883 
464 28. Finer, N., et al. (2000) Sibutramine is effective for weight loss and diabetic 465 control in obesity with type 2 diabetes: a randomised, double-blind, placebo466 controlled study. Diabetes Obes Metab 2, 105-112

467 29. Hansen, D.L., et al. (1998) Thermogenic effects of sibutramine in humans. 468 Am J Clin Nutr 68, 1180-1186

469 30. Torp-Pedersen, C., et al. (2007) Cardiovascular responses to weight 470 management and sibutramine in high-risk subjects: an analysis from the SCOUT 471 trial. Eur Heart J 28, 2915-2923

472 31. Kharitonenkov, A. (2009) FGFs and metabolism. Curr Opin Pharmacol 9, $473 \quad 805-810$

474 32. De Sousa-Coelho, A.L., et al. (2012) Activating transcription factor 4475 dependent induction of FGF21 during amino acid deprivation. Biochem J 443, $476 \quad 165-171$

477 33. Fisher, F.M., et al. (2012) FGF21 regulates PGC-1alpha and browning of 478 white adipose tissues in adaptive thermogenesis. Genes Dev 26, 271-281

479 34. Hondares, E., et al. (2011) Thermogenic activation induces FGF21 480 expression and release in brown adipose tissue.J Biol Chem 286, 12983-12990

481 35. Hondares, E., et al. (2010) Hepatic FGF21 expression is induced at birth 482 via PPARalpha in response to milk intake and contributes to thermogenic 483 activation of neonatal brown fat. Cell Metab 11, 206-212

484 36. Coskun, T., et al. (2008) Fibroblast growth factor 21 corrects obesity in 485 mice. Endocrinology 149, 6018-6027

486 37. Fisher, F.M., et al. (2010) Obesity is a fibroblast growth factor 21 (FGF21)487 resistant state. Diabetes 59, 2781-2789 
38. Ogawa, Y., et al. (2007) BetaKlotho is required for metabolic activity of 489 fibroblast growth factor 21. Proc Natl Acad Sci U S A 104, 7432-7437

490 39. Whittle, A.J., et al. (2012) BMP8B Increases Brown Adipose Tissue 491 Thermogenesis through Both Central and Peripheral Actions. Cell 149, 871-885

492 40. Sieber, C., et al. (2009) Recent advances in BMP receptor signaling. 493 Cytokine Growth Factor Rev 20, 343-355

494 41. Lopez, M., et al. (2010) Hypothalamic AMPK and fatty acid metabolism 495 mediate thyroid regulation of energy balance. Nat Med 16, 1001-1008

496 42. Fournier, B., et al. (2012) Blockade of the activin receptor IIb activates 497 functional brown adipogenesis and thermogenesis by inducing mitochondrial 498 oxidative metabolism. Mol Cell Biol 32, 2871-2879

499 43. Morrison, S.F., et al. (2012) Central control of brown adipose tissue 500 thermogenesis. Front Endocrinol (Lausanne) 3

501 44. Pingle, S.C., et al. (2007) Capsaicin receptor: TRPV1 a promiscuous TRP 502 channel. Handb Exp Pharmacol, 155-171

503 45. Kawada, T., et al. (1986) Capsaicin-induced beta-adrenergic action on 504 energy metabolism in rats: influence of capsaicin on oxygen consumption, the 505 respiratory quotient, and substrate utilization. Proc Soc Exp Biol Med 183, 250506256

507 46. Yoneshiro, T., et al. (2012) Nonpungent capsaicin analogs (capsinoids) 508 increase energy expenditure through the activation of brown adipose tissue in 509 humans. Am J Clin Nutr 95, 845-850

510 47. Ma, S., et al. (2012) Activation of the cold-sensing TRPM8 channel triggers 511 UCP1-dependent thermogenesis and prevents obesity. J Mol Cell Biol 4, 88-96 
512 48. Feldmann, H.M., et al. (2009) UCP1 ablation induces obesity and abolishes

513 diet-induced thermogenesis in mice exempt from thermal stress by living at

514 thermoneutrality. Cell Metab 9, 203-209

515 49. Mano-Otagiri, A., et al. (2010) Genetic suppression of ghrelin receptors 516 activates brown adipocyte function and decreases fat storage in rats. Regul Pept $517 \quad 160,81-90$

518 50. Mano-Otagiri, A., et al. (2009) Ghrelin suppresses noradrenaline release in

519 the brown adipose tissue of rats. J Endocrinol 201, 341-349

520 51. Zhang, Z., et al. (2011) Neuronal receptor activity-modifying protein 1

521 promotes energy expenditure in mice. Diabetes 60, 1063-1071

522 52. Jordan, S.D., et al. (2010) Sensing the fuels: glucose and lipid signaling in 523 the CNS controlling energy homeostasis. Cell Mol Life Sci 67, 3255-3273

524 53. Karnani, M.M., et al. (2011) Activation of central orexin/hypocretin 525 neurons by dietary amino acids. Neuron 72, 616-629

526 54. Rusyniak, D.E., et al. (2008) When administered to rats in a cold 527 environment, 3,4-methylenedioxymethamphetamine reduces brown adipose 528 tissue thermogenesis and increases tail blood flow: effects of pretreatment with 529 5-HT1A and dopamine D2 antagonists. Neuroscience 154, 1619-1626

530 55. Moukaddem, M., et al. (1997) Increase in diet-induced thermogenesis at 531 the start of refeeding in severely malnourished anorexia nervosa patients. $\mathrm{Am} J$ 532 Clin Nutr 66, 133-140

533 56. Allison, D.B., et al. (1999) Antipsychotic-induced weight gain: a 534 comprehensive research synthesis. Am J Psychiatry 156, 1686-1696 
535 57. Seeman, P., et al. (2005) Dopamine supersensitivity correlates with

536 D2High states, implying many paths to psychosis. Proc Natl Acad Sci U S A 102, $537 \quad 3513-3518$

538 58. Bymaster, F.P., et al. (1996) Radioreceptor binding profile of the atypical 539 antipsychotic olanzapine. Neuropsychopharmacology 14, 87-96

540 59. Madden, C.J., and Morrison, S.F. (2010) Endogenous activation of spinal 5-

541 hydroxytryptamine (5-HT) receptors contributes to the thermoregulatory

542 activation of brown adipose tissue. Am J Physiol Regul Integr Comp Physiol 298, 543 R776-783

544 60. Ferguson, J.M., and Feighner, J.P. (1987) Fluoxetine-induced weight loss in 545 overweight non-depressed humans. Int J Obes 11 Suppl 3, 163-170

546 61. Connoley, I.P., et al. (1999) Thermogenic effects of sibutramine and its 547 metabolites. Br J Pharmacol 126, 1487-1495

548 62. Tseng, Y.H., et al. (2010) Cellular bioenergetics as a target for obesity 549 therapy. Nat Rev Drug Discov 9, 465-482

550 63. Wijers, S.L., et al. (2009) Recent advances in adaptive thermogenesis:

551 potential implications for the treatment of obesity. Obes Rev 10, 218-226

552 64. Crisan, M., et al. (2008) A reservoir of brown adipocyte progenitors in 553 human skeletal muscle. Stem Cells 26, 2425-2433

554 65. Stowell, K.M. (2008) Malignant hyperthermia: a pharmacogenetic 555 disorder. Pharmacogenomics 9, 1657-1672

556 66. Ukropec, J., et al. (2006) Leptin is required for uncoupling protein-1557 independent thermogenesis during cold stress. Endocrinology 147, 2468-2480 
558 67. Ketzer, L.A., et al. (2009) Cardiac sarcoplasmic reticulum Ca2+-ATPase:

559 heat production and phospholamban alterations promoted by cold exposure and

560 thyroid hormone. Am J Physiol Heart Circ Physiol 297, H556-563

561 68. Mahmmoud, Y.A., and Gaster, M. (2012) Uncoupling of sarcoplasmic

562 reticulum $\mathrm{Ca}(2)(+)$-ATPase by $\mathrm{N}$-arachidonoyl dopamine. Members of the 563 endocannabinoid family as thermogenic drugs. Br J Pharmacol 166, 2060-2069

564 69. Bal, N.C., et al. (2012) Sarcolipin is a newly identified regulator of muscle-

565 based thermogenesis in mammals. Nat Med 18, 1857

566

567 